\title{
LOCALLY COMPACT FULL HOMEOMORPHISM GROUPS ARE ZERO-DIMENSIONAL
}

\author{
JAMES KEESLING
}

\begin{abstract}
Let $X$ be a metric space and let $G(X)$ be the space of homeomorphisms of $X$ with the compact open topology.

THEOREM. If $G(X)$ is locally compact, then it is zero-dimensional.

Some examples are also given of metric spaces $X$ for which $G(X)$ is compact.
\end{abstract}

Introduction. Let $X$ be a metric space and $G(X)$ be the set of all homeomorphisms of $X$ onto $X$ endowed with the compact open topology. In [9, Theorem 7] de Groot showed that algebraically $G(X)$ may be any group. In [7] Cook showed that $G(X)$ may be topologically a Cantor set with $X$ a metric continuum. In [5] Brechner showed that $G(X)$ may be algebraically and topologically any countable infinite product of finite cyclic groups with $X$ a onedimensional Peano continuum. The main theorem of this paper shows that the zero-dimensionality of the examples in [5] and [7] is an essential feature. We prove that if $G(X)$ is locally compact, then it is zero-dimensional. In contradistinction to this result it is known that $G(X)$ may be one-dimensional or infinite-dimensional [6]. The principal tool in our proof is the work of Beck in [3]. A function space result is also proved in this paper which improves some of the results in [4] and [8]. This result is then used in the proof of the main theorem. In the last section of the paper it is shown that for any cardinal $\alpha, G(X)$ may be topologically and algebraically $Z_{2}^{\alpha}$ where $Z_{2}$ is the discrete group with two elements.

Preliminaries. Throughout the paper let $X$ and $Y$ be regular $T_{1}$ topological spaces and let $Y^{X}$ denote the set of all continuous functions from $X$ into $Y$. For a set $A \subset X$ and $B \subset Y$ let $(A, B)$ be the set of all functions $f \in Y^{X}$ such that $f(A) \subset B$. We topologize $Y^{X}$ by taking as a subbase all sets of the form $(K, V)$ where $K \subset X$ is compact and $V \subset Y$ is open. The topology so defined is called the compact open topology or c-topology as in $\left[10\right.$, p. 257]. Of course $G(X) \subset X^{X}$, but $G(X)$ may not be a topological group in the $c$-topology. In [2] Arens

Received by the editors March 26, 1970.

AMS 1970 subject classifications. Primary 57E05; Secondary 54C35.

Key words and phrases. Function space, compact open topology, space of homeomorphisms, flow, topological group, one parameter subgroup, locally compact topological group, zero-dimensional. 
gives conditions on $X$ under which $G(X)$ is a topological group. If $H$ is a subgroup of $G(X)$, then $H$ may be a topological group even though $G(X)$ is not. We show that if $H$ is locally compact, then $H$ is a topological group which improves results in [8] and [4, pp. 305-308]. For background in function spaces see [4], [10], and [13].

By a flow on $X$ is meant a topological group action of the additive reals $F: R \times X \rightarrow X$. The invariant set of the flow $F$ is the set $S=\{x \in X: F(t, x)=x$ for all $t \in R\}$.

If $h$ is a homeomorphism of $X$ onto $X$, then the support of $h$ is the set supp $h=\{x \in X: h(x) \neq x\}$. Clearly supp $h$ is an open set.

1. A function space result. We now show that if $H$ is a subgroup of $G(X)$ and locally compact in the $c$-topology, then $H$ is a topological group. It is also shown that if $X$ is a $k$-space, then the evaluation map is an effective action of $H$ on $X$.

Lemмa 1. If $C$ is a compact set of functions in $Y^{x}$ and $K$ is a compact set in $X$, then $Q=\{f(x): f \in C$ and $x \in K\}$ is compact.

Proof. Let $\left\{U_{\alpha}: \alpha \in A\right\}$ be a cover of $Q$ by open sets of $Y$. Let $F(A)$ be the set of finite subsets of $A$. For $B \in F(A)$ define $U(B)$ $=\bigcup_{\alpha \in B} U_{\alpha}$. Now if $f \in C$, then by the continuity of $f, f[K]$ is compact. Since $f[K] \subset Q$ there must be a $B \in F(A)$ such that $f[K]$ $\subset U(B)$. Thus $C \subset \bigcup_{B \in F(A)}(K, U(B))$. Since $C$ is compact, there is a finite collection $\left\{B_{1}, \cdots, B_{n}\right\}$ in $F(A)$ such that $C \subset \bigcup_{i=1}^{n}\left(K, U\left(B_{i}\right)\right)$. If we let $A^{\prime}=\bigcup_{i=1}^{n} B_{i}$, then one can easily show that $Q \subset \bigcup_{\alpha \in A^{\prime}} U_{\alpha}$. This shows the compactness of $Q$.

Lemma 2. Let $C$ be a compact subset of $Y^{X}$ and $T: Z^{Y} \times Y^{X} \rightarrow Z^{X}$ be defined by $T(g, f)=g \circ f$. Then $T \mid Z^{Y} \times C$ is continuous.

Proof. Let $g \in Z^{Y}$ and $f \in Y^{X}$. Let $K \subset X$ be compact and $V \subset Z$ be open such that $g \circ f \in(K, V)$. Let $O$ be open in $Y$ such that $f[K]$ $\subset O \subset \bar{O} \subset g^{-1}(V)$. Now $(K, \bar{O}) \subset Y^{X}$ is a closed set in the function space. Let $C^{\prime}=C \cap(K, \bar{O})$. Then $C^{\prime}$ is compact. Let $Q$ $=\left\{h(x): x \in K\right.$ and $\left.h \in C^{\prime}\right\}$. By Lemma $1, Q$ is compact. Now $(K, O)$ $\cap C$ is a neighborhood of $f$ in $C$ and $(Q, V)$ is a neighborhood of $g$ in $Z^{Y}$ with $T(q, h) \in(K, V)$ for all $h \in(K, O) \cap C$ and all $q \in(Q, V)$. Therefore $T \mid Z^{Y} \times C$ is continuous.

THEOREM 1. If $H$ is a subgroup of $G(X)$ and locally compact in the $c$-topology, then $H$ is a topological group. If in addition $X$ is a k-space, then $H$ acts effectively on $X$.

Proof. By Lemma 2, composition is continuous on $H$ and thus $H$ is 
a topological semigroup. A locally compact topological semigroup which is also an algebraic group is a topological group by a theorem of Ellis [11]. Thus $H$ is a topological group. For the last part of the theorem, if $X$ is a $k$-space, then so is $H \times X$. Thus the evaluation map $\omega(h, x)=h(x)$ is continuous on $H \times X$ to $X$ by [10, Corollary $3.2, \mathrm{p}$. 261]. This is an effective group action of $H$ on $X$.

REMARK. It is well known that if $F: G \times X \rightarrow X$ is an effective action of the compact group $G$ on $X$, then the natural map $\hat{F}: G \rightarrow G(X)$ is an imbedding of $G$ in $G(X)$. So one may study effective actions of compact groups on $X$ by studying groups of homeomorphisms of $X$ which are compact in the $c$-topology. However, one cannot do this for locally compact groups $G$. A locally compact group $G$ may act effectively on $X$ by $F: G \times X \rightarrow X$ in such a way that the natural map $\hat{F}: G \rightarrow G(X)$ is not an imbedding. An example of this is an irrational flow on the torus.

REMARK. Theorem 1 shows that there is an error in $[8$, p. 675] where Dieudonné essentially claims that $H$ may be locally compact in the $c$-topology and yet not a topological group.

2. A remark about flows. The very elegant result of Beck [3] is used in the proof of our main result. However, the statement of his theorem is not as strong as we would like it to be. One can easily verify that Beck actually proved the following theorem.

Theorem 2 (BeCK). Let $X$ be a metric space and $F: R \times X \rightarrow X$ be a flow on $X$ with invariant set $S$. Then for any closed set $S^{\prime}$ containing $S$ one can construct a new flow $F^{\prime}: R \times X \rightarrow X$ whose invariant set is $S^{\prime}$. Moreover, for any $x \in X-S^{\prime}$ with orbit $O(x)$ under $F$, the orbit of $x$ under $F^{\prime}$ is just the set of points which can be joined to $x$ by an arc in $O(x)-S^{\prime}$.

3. The main result. We need the following two technical lemmas before proving our main theorem.

Lemma 3. Let $x \in X$ and let $\mathfrak{u}$ be a neighborhood of the identity $e$ in $G(X)$ in the c-topology. Then there is a neighborhood $U$ of $x$ in $X$ such that $\{h \in G(X):$ supp $h \subset U\} \subset \mathcal{u}$.

Proof. We may take $u=\bigcap_{i=1}^{n}\left(K_{i}, V_{i}\right) \cap G(X)$ where $K_{i}$ is compact and $V_{i}$ is open in $X$ with $K_{i} \subset V_{i}$. We may also suppose that if $x \notin K_{i}$, then $x \notin \bar{V}_{i}$. Let $U=\left(\bigcap_{x \in \nabla_{i}} V_{i}\right) \cap\left(\bigcap_{x \notin K_{i}} X-\bar{V}_{i}\right)$. Then $x \in U$ and $U$ is open in $X$. Let $g \in G(X)$ have its support in $U$. Let $y \in K_{i}$. Then if $y \notin U$, then $g(y)=y \in V_{i}$. On the other hand, if $y \in U$, then $x \in V_{i}$. Thus $U \subset V_{i}$. Since $g(y) \in U, g(y) \in V_{i}$. This implies that $g \in \bigcap_{i=1}^{n}\left(K_{i}, V_{i}\right)$. 
Lemma 4. Suppose that $\left\{g_{i}\right\}$ is a collection of homeomorphisms of $X$ with $\left\{x_{i}\right\}$ and $\left\{y_{i}\right\}$ subsets of $X$ satisfying:

(1) $g\left(x_{i}\right)=y_{i}$,

(2) $\operatorname{supp} g_{i} \cap \operatorname{supp} g_{j}=\varnothing$ for all $i \neq j$,

(3) $\mathrm{Cl}\left\{x_{i}\right\} \cap \mathrm{Cl}\left\{y_{i}\right\}=\varnothing$, and

(4) $\mathrm{Cl}\left\{x_{i}\right\}$ is compact.

Then $\left\{g_{i}\right\}$ is closed and discrete in the function space $G(X)$ in the ctopology.

Proof. Suppose that $g$ is a limit point of $\left\{g_{i}\right\}$ in $G(X)$. Then let $x_{j} \in V$ with $V$ open and $\bar{V} \cap \mathrm{Cl}\left\{y_{i}\right\}=\varnothing$. There is only one $g_{i}$ such that $g_{i}\left(x_{j}\right) \notin \bar{V}$, namely $g_{j}$. If $g\left(x_{j}\right) \notin \bar{V}$, then $g$ is not a limit point of the set $\left\{g_{i}\right\}$. Thus $g\left(x_{j}\right) \in \bar{V}$ for every neighborhood $V$ of $x_{j}$. This implies that $g\left(x_{j}\right)=x_{j}$ for all $j$. Therefore $g \mid \mathrm{Cl}\left\{x_{i}\right\}$ is the identity. Let $K=\mathrm{Cl}\left\{x_{i}\right\}$ and $K \subset V \subset \bar{V} \subset X-\mathrm{Cl}\left\{y_{i}\right\}$ with $V$ open in $X$. Then $(K, V)$ is a neighborhood of $g$ containing no $g_{i}$, a contradiction. Therefore the set $\left\{g_{i}\right\}$ has no limit points and is closed and discrete in $G(X)$.

LEMMA 5. If $G$ is a nontrivial connected locally compact topological group, then $G$ has a nontrivial one parameter subgroup.

Proof. This is well known. See for instance p. 175 and Theorem 1, p. 192, of [14].

TheOREm 3. If $X$ is a metric space and $G(X)$ is locally compact, then $\operatorname{dim} G(X)=0$.

Proof. Let us first observe that we may assume that $X$ does not contain any free arcs, that is, any arcs whose interiors are open in the space. If there is a free arc $[a, b]$ in $X$ whose interior $(a, b)$ is open in $X$, then letting $H=\{h \in G(X): h \mid X-(a, b)$ is the identity $\}$ we get that $H$ is a closed subgroup of $G(X)$ which is not locally compact. Actually $H$ is homeomorphic to separable infinite-dimensional Hilbert space [1]. So, assume that $X$ has no free arcs. Now assume that $G(X)$ is locally compact and not zero-dimensional. Then $G(X)$ is a topological group by Theorem 1 . In a locally compact topological group zero-dimensionality is equivalent to total disconnectedness, since this is true for locally compact paracompact spaces and such a group is paracompact [12, Theorem 8.13, p. 76]. Thus the component of the identity, $G_{0}$, of $G(X)$ is not trivial. Thus $G_{0}$ is a nontrivial connected locally compact topological group. By Lemma $5, G_{0}$ contains a nontrivial one parameter subgroup $f: R \rightarrow G_{0}$. Since $X$ is a $k$-space [10, 9.3 , p. 248] we get that $F(t, x)=f(t)(x)$ is continuous from $R \times X$ to $X$ 
and is a nontrivial flow on $X$ by Theorem 1 . Let $x$ be a point which has a nondegenerate orbit under the action of $F$. Let $u$ be an arbitrary neighborhood of $e$ in $G(X)$ and let $U$ be an open set in $X$ containing $x$ such that if supp $g \subset U$, then $g \in \mathcal{U}$ as in Lemma 3 . We also assume that no points of the invariant set of $F$ are in $U$. Our plan now is to construct a countably infinite set of homeomorphisms $\left\{g_{i}\right\}_{i=1}^{\infty}$ such that supp $g_{i} \subset U$ for all $i$ with $\left\{g_{i}\right\}_{i=1}^{\infty}$ closed and discrete in $G(X)$. By the choice of $U,\left\{g_{i}\right\}_{i=1}^{\infty} \subset \mathcal{U}$ and thus $\mathcal{u}$ cannot have compact closure in $G(X)$.

Claim. There is a set of homeomorphisms of $X,\left\{g_{i}\right\}_{i=1}^{\infty}$, such that supp $g_{i} \subset U$ for all $i$ with $\left\{g_{i}\right\}_{i=1}^{\infty}$ closed and discrete in $G(X)$.

Proof of Claim. Let $\tau$ be a small enough positive real number that $A=\{F(t, x):-2 \tau \leqq t \leqq 2 \tau\}$ is contained in $U$ and is a simple arc. Since $X$ has no free arcs, there is a sequence of points $x_{i}$ converging to $x$ such that $x_{i} \notin A$ for all $i$. Let $B=\{F(t, x):-\tau \leqq t \leqq \tau\}$ and $B_{i}$ $=\left\{F\left(t, x_{i}\right):-\tau \leqq t \leqq \tau\right\}$. Then $B_{i} \cap B=\varnothing$ for all $i$, for if $z \in B_{i} \cap B$, then $z=F(t, x)=F\left(t^{\prime}, x_{i}\right)$ with $|t|,\left|t^{\prime}\right| \leqq \tau$ and $F\left(t-t^{\prime}, x\right)=x_{i}$. This would imply that $x_{i} \in A$ since $\left|t-t^{\prime}\right| \leqq 2 \tau$. Thus $B_{i} \cap B=\varnothing$. We claim now that there is a subsequence $\left\{B_{i_{j}}\right\}_{j=1}^{\infty}$ such that $B_{i j} \cap B_{i_{k}}=\varnothing$ for $j \neq k$ with $B_{i j} \subset U$ for all $j$. We construct such a subsequence inductively. By the continuity of $F$ the $B_{i}$ 's are finally in $U$. Let $B_{i_{1}}$ be such that for all $i \geqq i_{1}, B_{i} \subset U$. Suppose that there is a subsequence $\left\{B_{i_{j}}\right\}_{j=1}^{\infty}$ such that $B_{i_{j}} \cap B_{i_{1}} \neq \varnothing$ for all $j$. Let $z_{j} \in B_{i_{j}} \cap B_{i_{1}}$ and let $\left|t_{j}\right| \leqq \tau$ be such that $F\left(t_{j}, x_{i_{j}}\right)=z_{j}$. There is a subsequence of $\left\{t_{j}\right\}$ which converges to $t_{0}$ in $[-\tau, \tau]$. Call the subsequence $\left\{t_{j}\right\}$ also. By the continuity of $F$ we have $F\left(t_{j}, x_{i_{j}}\right)$ converges to $F\left(t_{0}, x\right)$. But $F\left(t_{j}, x_{i_{j}}\right) \in B_{i_{1}}$ for all $j$. Thus $F\left(t_{0}, x\right) \in B_{i_{1}}$ contradicting the fact that $B \cap B_{i_{1}}=\varnothing$. We have now shown that there exists an $i_{2}>i_{1}$ such that for $i \geqq i_{2}, B_{i} \cap B_{i_{1}}=\varnothing$. In a similar fashion get an $i_{3}>i_{2}$ such that for $i \geqq i_{3}, \quad B_{i} \cap B_{i_{2}}=\varnothing$. Continuing inductively we get a sequence $\left\{B_{i j}\right\}_{j=1}^{\infty}$ with $B_{i j} \subset U$ for all $j$ and $B_{i_{j}} \cap B_{i_{k}}=\varnothing$ for all $j \neq k$. Resubscript and call this subsequence $\left\{B_{i}\right\}_{i=1}^{\infty}$. Now let $\left\{U_{i}\right\}_{i=1}^{\infty}$ be a collection of open sets in $X$ satisfying

(1) $U_{i} \subset U$ for all $i$;

(2) $B_{i} \subset U_{i}$ for all $i$; and

(3) $U_{i} \cap U_{j}=\varnothing$ for $i \neq j$.

Such a collection of $U_{i}$ 's exists since the $B_{i}$ 's are disjoint and the set $F\left[[-\tau, \tau] \times\left\{x_{i}\right\} \cup\{x\}\right]$ is compact in $X$. Now let $F_{i}$ be a flow on $X$ having invariant set $X-U_{i}$ as in Theorem 2. By Theorem $2, B_{i}$ is a subset of the orbit of $x_{i}$ under the flow $F_{i}$. Thus there is a $t_{i} \in R$ such that $F_{i}\left(t_{i}, x_{i}\right)=F\left(\tau, x_{i}\right)$. Let $g_{i}$ be the homeomorphism of $X$ defined by $g_{i}(z)=F_{i}\left(t_{i}, z\right)$ for all $z \in X$. Then $g_{i}\left(x_{i}\right)=F\left(\tau, x_{i}\right)$. If we let $y_{i}$ $=g_{i}\left(x_{i}\right)$, then we note that $\mathrm{Cl}\left\{x_{i}\right\}=\left\{x_{i}\right\} \cup\{x\}$ is compact and 
$\mathrm{Cl}\left\{y_{i}\right\}=\left\{y_{i}\right\} \cup\{F(\tau, x)\}$. Thus $\mathrm{Cl}\left\{x_{i}\right\} \cap \mathrm{Cl}\left\{y_{i}\right\}=\varnothing$. Thus the collection $\left\{g_{i}\right\}_{i-1}^{\infty}$ satisfies the conditions of Lemma 4 and is closed and discrete in $G(X)$. Since supp $g_{i} \subset U_{i} \subset U$, this collection satisfies the conditions of the claim. We have now proved the claim and thus the theorem.

4. Examples of compact full groups of homeomorphisms. We now show that if $\alpha$ is any cardinal and $Z_{2}$ is the two element group $\{0,1\}$ with discrete topology and addition modulo two, then there is a metric space $X$ with $G(X)$ topologically and algebraically $Z_{2}^{\alpha}$.

Lemмa 6. For any cardinal $\alpha$ there is a collection of one-dimensional metric spaces $\left\{X_{\beta}: \beta \in B\right\}$ such that

(1) $|B|=\alpha$;

(2) for $\beta \neq \gamma$ in $B, X_{\beta}$ is not homeomorphic to $X_{\gamma}$; and

(3) the only homeomorphism $h: X_{\beta} \rightarrow X_{\beta}$ is the identity for all $\beta$ in $B$.

Proof. Let $\Gamma_{\beta}$ be a collection of infinite cardinals such that $\Gamma_{\beta}$ $=\bigcup_{i=1}^{\infty} \Gamma_{\beta, i}$ is a partition of $\Gamma_{\beta}$ with (1) $\Gamma_{\beta, 1}=\{\lambda\}$ a single cardinal and (2) $\left|\Gamma_{\beta, i+1}\right|=\sup \left\{\lambda: \lambda \in \bigcup_{j=1}^{i} \Gamma_{\beta, j}\right\}$. We also want the collection $\left\{\Gamma_{\beta}: \beta \in B\right\}$ to be pairwise disjoint. One can easily construct this collection by transfinite induction. Now construct $X_{\beta}$ in the following manner. Let $\lambda$ be a cardinal and $n$ an integer and let $S(\lambda, n)$ be $\lambda$ copies of $\left[0,2^{-n}\right]$ all joined at their zeros with the metric $d(x, y)$ $=|x-y|$ for $x$ and $y$ on the same branch of $S(\lambda, n)$ and $d(x, y)=x+y$ for $x$ and $y$ on distinct branches. Now let $X_{1}=S(\lambda, 1)$ where $\{\lambda\}$ $=\Gamma_{\beta, 1}$. Let $A_{1}$ be the central zero of $X_{1}$. Let $A_{2}$ be the midpoints of the branches of $X_{1}$. Then $\left|A_{2}\right|=\lambda=\left|\Gamma_{\beta, 2}\right|$. Let $f_{2}: A_{2} \rightarrow \Gamma_{\beta, 2}$ be a bijection. For $a \in A_{2}$ attach $S\left(f_{2}(a), 2^{-1}\right)$ to the point $a$ at its center. Let $X_{2}$ be $X_{1}$ union all of its new appendages $S\left(f_{2}(a), 2^{-1}\right)$ with metric $d_{2}$ defined by $d_{2}(x, y)=d_{1}(x, y)$ for $x, y \in X_{1} ; d_{2}(x, y)=d_{1}(x, a)+d(a, y)$ for $y$ $\in S\left(f_{2}(a), 2^{-1}\right)$ and $x \in X_{1}$; and $d_{2}(x, y)=d\left(x, a_{1}\right)+d_{1}\left(a_{1}, a_{2}\right)+d\left(y, a_{2}\right)$ for $x \in S\left(f_{2}\left(a_{1}\right), 2^{-1}\right)$ and $y \in S\left(f_{2}\left(a_{2}\right), 2^{-1}\right)$. Let $A_{3}$ be the midpoints of all free arcs in $X_{2}$ (this includes the segments $[0, a]$ and $[a, 1]$ in $X_{1}$ for $a \in A_{2}$ ). One can easily see that $\left|A_{3}\right|=\sup \left\{\lambda: \lambda \in \Gamma_{\beta, 1} \cup \Gamma_{\beta, 2}\right\}=\left|\Gamma_{\beta, 3}\right|$. Let $f_{3}: A_{3} \rightarrow \Gamma_{\beta, 3}$ be a bijection. For each $a \in A_{3}$ attach $S\left(f_{3}(a), 2^{-2}\right)$ to $a$ at its center and let $X_{3}$ be $X_{2}$ union its new appendages with metric $d_{3}$ on $X_{3}$ which extends the metric $d_{2}$ as in the previous step. Repeatng this process inductively we get an increasing sequence of metric spaces $X_{i} \subset X_{i+1}$ with metrics $d_{i}$ on $X_{i}$ such that $d_{i} \mid X_{j}=d_{j}$ for $j \leqq i$. Let $X=\bigcup_{i=1}^{\infty} X_{i}$ with metric $d(x, y)=d_{i}(x, y)$ for $x$ and $y$ in $X_{i}$. Then $d$ is well defined and $X$ is a metric space. Let $A=\bigcup_{i=1}^{\infty} A_{i}$ in the construction. Letting $f=\bigcup_{i=1}^{\infty} f_{i}$ we get a bijection $f: A \rightarrow \Gamma_{\beta}$. Now for $a \in A, a$ has cut point order $f(a)$ in $X$. Since the cardinals in $\Gamma_{\beta}$ are distinct, $f(a) \neq f(b)$ for $a \neq b$ in $A$. For all other points $x$ of $X$, the cut 
point order of $x$ is just one or two. Thus any homeomorphism $h$ of $X_{\beta}$ on to $X_{\beta}$ must take $A$ onto $A$ and be the identity restricted to $A$. Thus $h$ must be the identity on $X_{\beta}$ since $A$ is dense. Thus each $X_{\beta}$ satisfies (3) of the lemma. Since $\Gamma_{\beta} \cap \Gamma_{\gamma}=\varnothing$ for $\beta \neq \gamma$ in $B$, a similar argument shows that $X_{\beta}$ and $X_{\gamma}$ cannot be homeomorphic. The lemma is now proved.

THEOREM 4. Let $\alpha$ be a cardinal. Then there is a one-dimensional metric space $X$ such that $G(X)$ is topologically and algebraically $Z_{2}^{\alpha}$.

Proof. Let $\left\{X_{\beta}: \beta \in B\right\}$ be a collection of metric spaces satisfying (1), (2), and (3) of Lemma 6 for the cardinal $\alpha$. Let $Y$ be the free topological union of the $X_{\beta}$ 's and $X=\{0,1\} \times Y$. Let $f$ be a homeomorphism of $X$ onto itself. Then $f\left[\{0\} \times X_{\beta}\right]=\left\{n_{\beta}\right\} \times X_{\beta}$ for $n_{\beta}=0$ or $n_{\beta}=1$. Let $F: G(X) \rightarrow \Pi\{0,1\}_{\beta}$ be defined by $F(f)=\left(n_{\beta}\right)$. One can easily see that $F$ is a topological and algebraic equivalence.

\section{REFERENCES}

1. R. D. Anderson, Spaces of homeomorphisms of finite graphs (preprint).

2. R. F. Arens, Topologies for homeomorphism groups, Amer. J. Math. 68 (1946), 593-610. MR 8, 479.

3. A. Beck, On invariant sets, Ann. of Math. (2) 67 (1958), 99-103. MR 19, 1064.

4. N. Bourbaki, General topology. Part 2, Hermann, Paris; Addison-Wesley, Reading, Mass., 1966, Chapter 10. MR 34 \#5044b.

5. B. L. Brechner, Topological groups which are not full homeomorphism groups (preprint).

6. - On the dimension of certain spaces of homeomorphisms, Trans. Amer. Math. Soc. 121 (1966), 516-548. MR 32 \#4662.

7. H. Cook, Continua which admit only the identity mapping onto non-degenerate subcontinua, Fund. Math. 60 (1967), 241-249. MR 36 \#3315.

8. J. Dieudonné, On topological groups of homeomorphisms, Amer. J. Math. 70 (1948), 659-680. MR 10, 137.

9. J. de Groot, Groups represented by homeomorphism groups. I, Math. Ann. 138 (1959), 80-102. MR 22 \#9959.

10. J. Dugundji, Topology, Allyn and Bacon, Boston, Mass., 1966, Chapter 12. MR 33 \#1824.

11. R. Ellis, $A$ note on the continuity of the inverse, Proc. Amer. Math. Soc. 8 (1957), 372-373. MR 18, 745.

12. E. Hewitt and K. A. Ross, Abstract harmonic analysis. Vol. I: Structure of topological groups. Integration theory, group representations, Die Grundlehren der math. Wissenschaften, Band 115, Academic Press, New York; Springer-Verlag, Berlin, 1963. MR 28 \#158.

13. J. L. Kelley, General topology, Van Nostrand, Princeton, N. J., 1955, Chapter 7. MR 16, 1136.

14. D. Montgomery and L. Zippen, Topological transformation groups, Interscience, New York, 1955. MR 17, 383.

15. J. Nagata, Modern dimension theory, Bibliotheca Mathematica, vol. 6, Interscience, New York, 1965. MR 34 \#8380.

University of Florida, Gainesville, Florida 32601 